\title{
Malaria Slaides Segmentation
}

\author{
Ruaa N. Youns \\ Department of Computer \& Information Engineering \\ College of Electronics Engineering \\ University of Mosul \\ Mosul, Iraq
}

\begin{abstract}
The analysis of blood cells in a microscopic image can provide useful information about a person's health. In particular, the red blood cell analysis abnormalities can detect diseases this diseases is important such as malaria effectively. The images of blood, acquired by a microscope, which are connected with digital camera, are analyses by the computer to diagnosis or can be facilely. Transferred to clinical centers of liquid blood samples. Malaria is an infectious disease transmitted by mosquitoes of animals and humans caused by parasites (a species of microorganisms) of the Plasmodium genus. Infections begin through the bite of infected female mosquitoes, sometimes this infection leads to death, so you must find a way to help detect malaria. This document shows how to improve the image of microscopic and filter the not necessary parts, and identify the red blood cells in each image; each red blood cell is separated into a separate image.
\end{abstract}

\section{General Terms}

Image processing, image segmentation.

\section{Keywords}

Labeling; opening; closing; malaria; plasmodium.

\section{INTRODUCTION}

There are many human diseases caused by parasites and these diseases are fatal and non-fatal and include malaria. Malaria is a type of disease that leads to death in some developing countries. In 2015, Approximately 214 million cases of malaria were found, resulting in approximately 480,000 deaths. $90 \%$ of those cases were in Africa. [1]. Malaria can become an epidemic during the rainy season. Therefore, Therefore, there is a need to develop treatments to reduce mortality and in the case of a pandemic-like situation, it is very difficult to equip equipment that identifies malaria, especially in poor areas. There are many test groups, such as the main malaria test group, but the cost of these groups is very large and it is difficult to place them in each health unit. [2] The manual diagnosis of malaria is long and tedious and requires a trained and skilled technician. The technician takes 15 minutes to evaluate 100 cells. The automated analysis of the blood cell is stronger, improves accuracy, reduces power and human error and saves time. [3]. The cause of malaria is the primary parasites of the plasmodium gender. [4-5]. life cycle of malaria parasite's it is divided to four stages: merozees, cyclic phase turbines, mature algorithm development stages, and then schizonts or gametocytes of the red blood cells. [5]

\section{SYSTEM DETAILES}

The system is expounded with help of diagram, as shown in figure 1 .

\author{
Majid D. Youns, PhD \\ Department of Computer \& Information Engineering \\ College of Electronics Engineering
Cont \\ Ninevah University \\ Mosul, Iraq
}

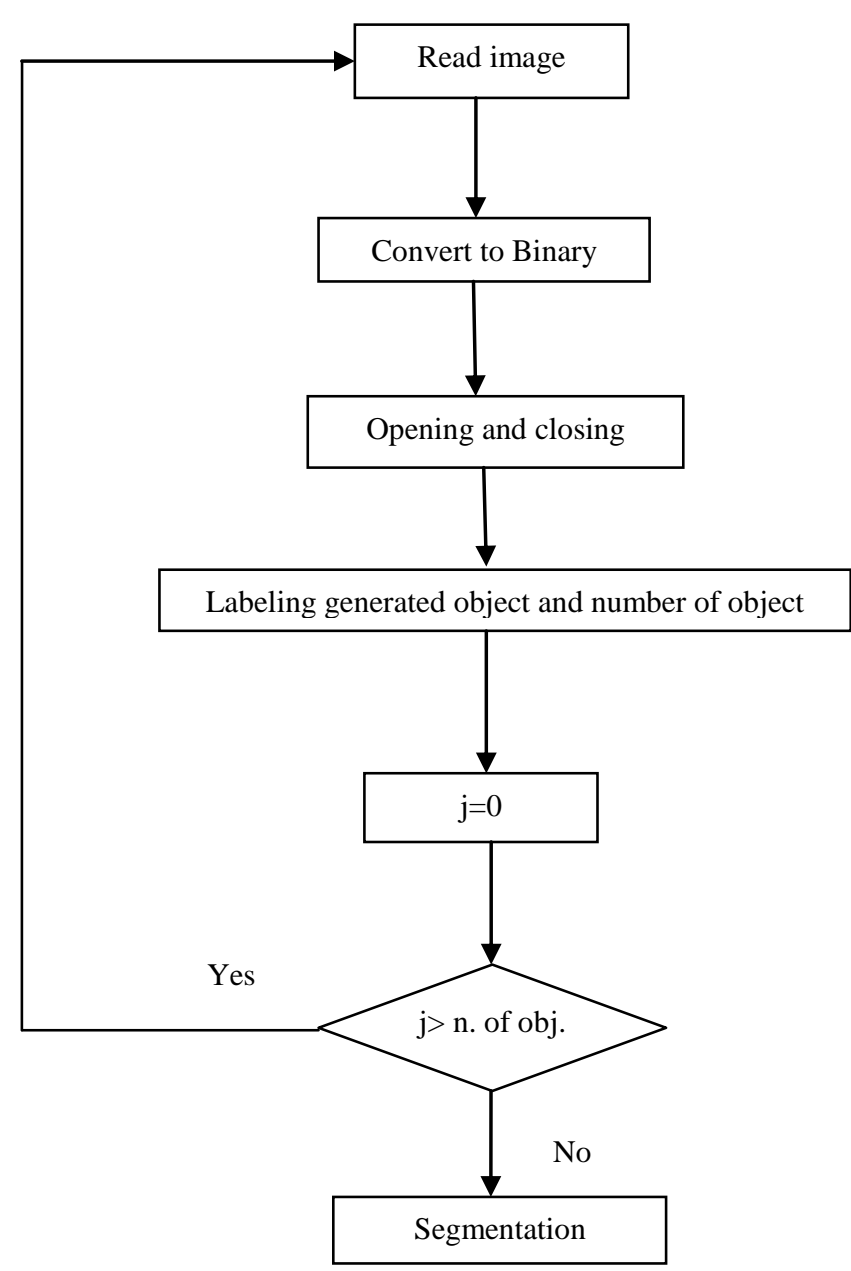

Fig 1: System block diagram

\section{IMAGE ACQUISITION}

Image acquisition it is first step for the system. Digital images for blood samples are obtained on slides by a camera that is installed on the microscope. As shown in figure 2. [2] 


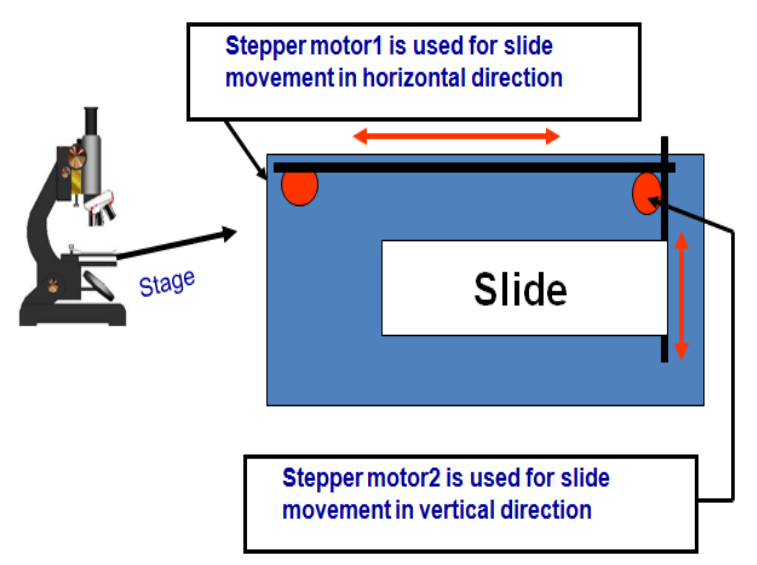

Fig 2: Slide control with stepper motor

After this the image converts to binary image by taking the global threshold for gray image as shown in figure 3 .

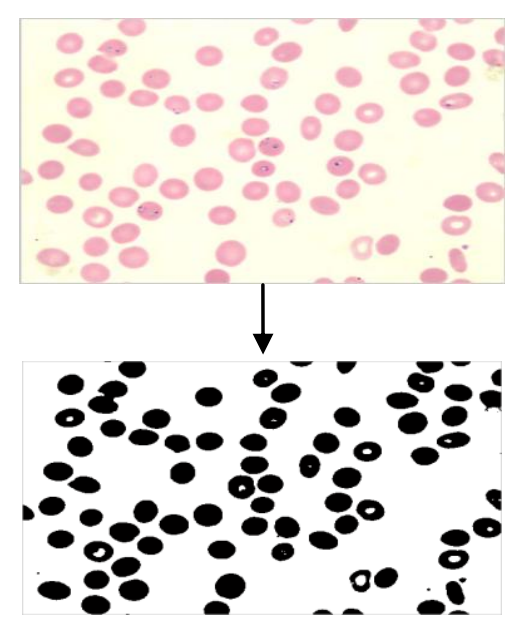

Fig 3: Binary image converting

\section{IMAGE ENHANCMENT}

The binary image is enhance by using opening and closing its remove unnecessary or small contours.

\subsection{Closing}

Image closing is also a process of compatibility of erosion and dilation. It differs from the opening process in Feeling the order of occurrence of corrosion and dilation Operation. Close image A by structuring element B Known in equation 1 .

$$
\text { A.B }=(\mathrm{A} \oplus \mathrm{B}) \ominus \mathrm{B}
$$

The relationship between erosion and dilation with closure is contained in the above mathematic statement. It shows that the closing operation is a dilation of image A before Element $\mathrm{B}$ structure and the result is eroded with the same structural element. The closed image limits are the points in structural element $\mathrm{B}$ that reach the maximum points of limits when "B" is "A" outside its limits. The closing process although smooth the parts of the profiles it generally blends tight dividers and thin gaps. As a result, it removes small holes and fills gaps within objects as shown in figure 4. [6] [7]

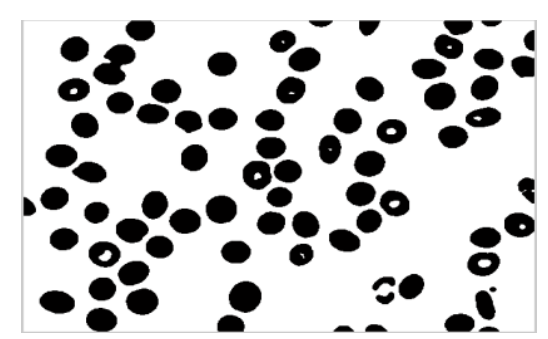

Fig 4: Binary image after closing

\subsection{Opening}

Open the image is a merge operation Erosion and dilation. Open $\mathrm{A}$ is defined by structuring element $\mathrm{B}$ in equation 2 .

$$
\mathrm{A} \text { о } \mathrm{B}=(\mathrm{A} \ominus \mathrm{B}) \oplus \mathrm{B} \ldots \ldots
$$

The above definition gives the relationship between opening, erosion and dilation. It states that the opening process is only image erosion by a structural element and the output is expanded using the same structural element. The boundaries of the open image are the points in structural element B, which reaches the extremes of boundary A as "rolled" around these limits. The Union's Literary Process Group is also used to find Points from the open image. The opening process enjoys the outline of an object Clear narrow bridges and also eliminates minor accessories exists in the object as shown in figure 5. [6] [7]

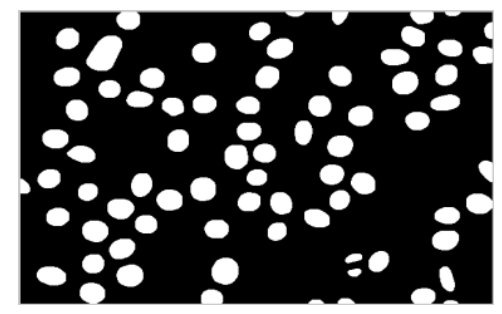

Fig 5: Inverted image after opening

\subsection{Dilation}

The dilation process makes an organism grow by size. The extent to which it grows depends on the nature and form of the structure element. Stretch a picture (set) by Structure element $\mathrm{B}$ is defined in equation 3.

$\mathrm{A} \oplus \mathrm{B}=\left\{\mathrm{z} \mid\left(\mathrm{B}^{\wedge}\right)_{\mathrm{z}} \cap \mathrm{A} \neq \varnothing\right\}-\ldots-\ldots-\ldots$

If group $B$ is reflected in its origin and transformed by $z$, the amplitude of $A$ to $B$ is the sum of all $z$ displacements $B \wedge$ and A contains at least one common element. Dilation, he said above, it adds pixels to the border elements. The dilation increments the number of pixels in the first value (foreground) and reduces the number of pixels in the zero value (background). The dilation process is used primarily to fill holes (missing pixels) in a continuous object. The process of dilation, because it adds pixels to the boundaries of the object and affect the intensity of that location, and as a result the effect of opacity can be observed. So it can be said that it is similar to filter low-pass filters used in the linear filtration of the image. [6] [7] [8]

\subsection{Erosion}

The erosion process is an extension of the expansion Process in context with process effect. This is erosion the process causes the object to lose its size. Each image is defined by element $\mathrm{B}$ as the organization structure in equation 4. 
$\mathrm{A} \ominus \mathrm{B}=\left\{\mathrm{z} \mid(\mathrm{B})_{\mathrm{z}} \subseteq \mathrm{A}\right\} \ldots \ldots$

Erosion of image A by element $\mathrm{B}$ structuring is group all $\mathrm{z}$ points so that element $\mathrm{B}$ is translated by $\mathrm{z}$ is a subset of the image. This process leads to loss Border pixels of the object. Erosion increases the number of pixels of zero (background) and reduces the number of pixels with the first value (the foreground). The erosion process removes those smaller structures from the structure of the structural element. So it can be used to remove noisy "connection" between two things. Because unwanted pixels are "scanned", the net effect is the object's sharpness in the image. The process of erosion is similar to sharpening a high pass filter that is used in linear filter image. [6] [7] [8]

\subsection{Labeling}

Labeling of connected components in a binary image is one of the most fundamental operations in pattern analysis, pattern recognition, computer vision, and machine intelligence. [9] Component connection labeling is used to visualize the computer to detect contiguous areas in binary digital images, although color images and data with high dimensions can also be processed. [10][11] When integrated into an image recognition system or interface between human and computer, component tags can be linked to operate on a variety of information. [12][13] The extraction of the point on the binary image resulting from a boundary step is usually done, but can be applied to gray and small images as well. Points may be calculated, filtered, and tracked. After opening and closing make a labeling to find the $\mathrm{RBC}$ and the number of $\mathrm{RBC}$ for each image the neighbors that used it are 4 because they removed the noise in the previous Stage. The size of the image is $40 * 40$ pixels the size was selected depending on the manual calculations where the radius of the red blood cell is approximately 20 so the size of the image was chosen, then all the coordinates of the red blood cells are found and then the center is calculated for each red blood cell. A mask is then made to cut off each red blood cell and store it as an independent image as shown in figure 6, the character under each image represent the code of this image. The mask is an image the size of this image is the same size of the original image and contains a circle the radius of this circle corresponds to the radius of the red blood cell, the center of the circle is variable according to the center of each red blood cell. In this algorithm the red blood cells on the edges of the image are incomplete and are not included in calculations because sometimes their radius is negative. Show the number of real cells in the image and the number of cells detected by the program in the table 1 .
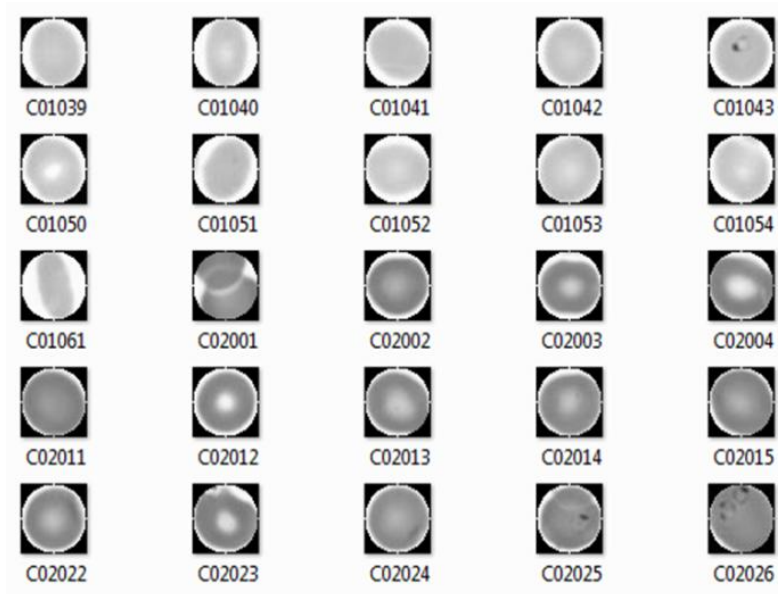

Fig 6: Examples of the extracted each $\mathrm{RBC}$ as an independent image

Table 1. Shows the number of real cells in the image and the number of cells detected by the program.

\begin{tabular}{|c|c|c|c|c|}
\hline $\begin{array}{c}\text { Test } \\
\text { images }\end{array}$ & 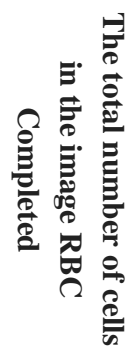 & 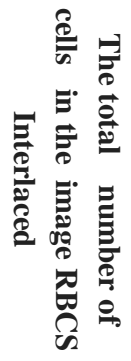 & 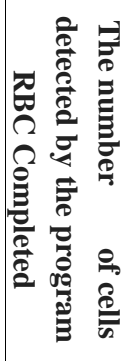 & 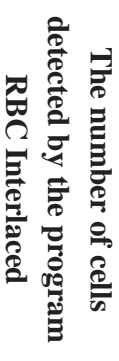 \\
\hline $\begin{array}{c}\text { Test } \\
\text { image } 1\end{array}$ & 60 & 1 & 60 & 2 \\
\hline $\begin{array}{c}\text { Test } \\
\text { image } 2\end{array}$ & 67 & 5 & 69 & 4 \\
\hline $\begin{array}{c}\text { Test } \\
\text { image } 3\end{array}$ & 65 & 4 & 56 & 6 \\
\hline $\begin{array}{c}\text { Test } \\
\text { image } 4\end{array}$ & 63 & 0 & 62 & 1 \\
\hline
\end{tabular}

\section{CONCLOSION}

In this stage, the image was split into several images. Each image contains one blood cell. In order to create our own data base with accuracy $99.7 \%$, the next step will be to insert the images in the database into another system such as the Neural Network or the Fuzzy Logic. 


\section{REFERENCES}

[1] Lu'is, R., Jos'e, M., Correia, d. C. , Dirk, E., Jaime S. C. 2016. Automated detection of malaria parasites on thick blood smears via mobile devices. International Conference On Medical Imaging Understanding and Analysis.

[2] Pramit, G., Debotosh, B., Mita N.and Dipak, K. B. Medical Aid for Automatic Detection of Malaria.

[3] Muhammad, I. R. 2015. automatic detection and classification of malarial parasite . International Journal of Biometrics and Bioinformatics (IJBB), Volume (9) : Issue (1)

[4] Courosh, M. et. all . Computer-Automated Malaria Diagnosis and Quantitation.

[5] Arash, M. , Tooraj, A., Morteza, I. 2013. Automatic Malaria Diagnosis System.

[6] Ravi, S., A M Khan, 2013. Morphological Operations for Image Processing

[7] Rafael G. ,digital image processing.
[8] Megha Goyal. 2011. Morphological Image Processing . IJCST Vol. 2, Iss ue 4, Oct . - Dec...

[9] Lifeng, H., Yuyan, C., Kenji, S. 2012. A New Two-Scan Algorithm for Labeling connected Components in Binary Images . Proceedings of the World Congress on Engineering Vol II,

[10] Hannan, S. Markku T.1988. Efficient Component Labeling of Images of Arbitrary Dimension Represented by Linear Bintrees.

[11] Michael, B. D., Hannan, S., Markku, T. 1992. A General Approach to Connected-Component Labeling for Arbitrary Image Representations

[12] Weijie, C., Maryellen L. G., Ulrich, B. 2006. A Fuzzy CMeans (FCM)-Based Approach for Computerized Segmentation of Breast Lesions in Dynamic ContrastEnhanced MR Images1 .

[13] Kesheng, W. ,Wendy K., Jacqueline, C. and Arie S., Using Bitmap Index for Interactive Exploration of Large Datasets. U.S. Department of Energy under Contract No. DE-AC03-76SF00098. 